\title{
Standardization of Spacing and Soil Volume Wetting for Drip Irrigation in Papaya (Carica papaya L.)
}

\author{
Manjunath B.L., Nair A.K. and Laxman R.H. \\ ICAR-Indian Institute of Horticultural Research, Bengaluru - 560 089, India \\ Email :blmanjunathagri@gmail.com
}

\begin{abstract}
Field experiments in two crops of papaya were conducted at ICAR-Indian Institute of Horticultural Research for four years during 2016-19 to standardise spacing with optimum soil volume wetting for drip irrigation. Narrowing the plant rows drastically reduced the plant height while leaf production affected significantly due to reduction in intra row spacing. The height at first fruiting was significantly lower with a spacing of $1.8 \mathrm{~m} \times 1.5 \mathrm{~m}(56.4$ cm) significantly differing from both $1.5 \mathrm{~m} \times 1.5 \mathrm{~m}(60.9 \mathrm{~cm})$ or $1.8 \mathrm{~m} \times 1.8 \mathrm{~m}(66.8 \mathrm{~cm})$. Significantly higher mean fruit yield $(42.2 \mathrm{t} / \mathrm{ha})$ was recorded with the spacing of $1.5 \mathrm{~m} \mathrm{x}$ $1.5 \mathrm{~m}$ as compared to either $1.8 \mathrm{~m} \times 1.5 \mathrm{~m}(23.4 \mathrm{t} / \mathrm{ha})$ or $1.8 \mathrm{~m} \times 1.8 \mathrm{~m}(22.1 \mathrm{t} / \mathrm{ha})$. Significantly higher water use efficiency $(71.3 \mathrm{~kg} / \mathrm{ha} . \mathrm{mm})$ was recorded in papaya by following closer spacing of $1.5 \mathrm{~m} \times 1.5 \mathrm{~m}$. Among the interactions, higher papaya yield $(48.0 \mathrm{t} / \mathrm{ha})$ was recorded with normal drip irrigation $(80 \%$ soil volume wetting) under closer spacing $(1.5 \mathrm{~m} . \times 1.5 \mathrm{~m})$. Further, higher water use efficiency $(129 \mathrm{~kg} / \mathrm{ha} . \mathrm{mm})$ could be obtained by scheduling the irrigation at $30 \%$ soil volume wetting especially by planting at $1.5 \mathrm{~m}$. $x 1.5 \mathrm{~m}$. spacing suggesting its suitability for water scarcity areas.
\end{abstract}

Key words: Papaya yield, Scheduling irrigation, Soil volume wetting, Spacing, Water use efficiency.

\section{INTRODUCTION}

Papaya is an important fruit crop cultivated in tropical and subtropical regions. Being a shallow rooted crop, papaya needs regular irrigation for its rapid growth and development. Further, the orchard should have a good drainage system and any amount of water logging will affect the growth. In papaya, stomata are found only on the abaxial leaf surface. They are sensitive to changes in the saturation deficit of the air. Stomata also respond quickly to changing light conditions. On clear days, midday suppression of photosynthesis occurs as a result of partial closure of the stomata (Carr, 2014).

A properly designed and operated drip irrigation system has to supply the water amount required by the crop and should also wet enough soil volume. Unlike surface and sprinkler irrigation, drip irrigation only wets part of the soil root zone. This may be as low as 30 per cent of the volume of soil wetted by the other methods. The wetting patterns which develop from dripping water onto the soil depend on discharge and soil type. Although only part of the root zone is wetted it is still important to meet the full water needs of the crop. Two of the key factors in the design of micro-irrigation systems to obtain the maximum benefits from these practices are the amount of water used and the volume of soil to be wetted.

The restricted volume of the wetted soil under drip irrigation and depth-width dimensions of this volume are of considerable practical importance. The volume of the wetted soil represents the amount of soil water stored in the root zone, its depth dimension should coincide with the depth of the root system while its width dimension should be related to the spacing between the emitters and lines. The parameters which influence the wetted soil volume are the available water holding capacity of the soil and the peak daily crop water use representing specific field conditions. The irrigation interval and the management-allowed deficit are additional parameters which affect the wetted volume and could be changed depending on 
crop sensitivity as well as water and irrigation equipment accessibility. A truncated ellipsoid is assumed to best represent the geometry of the wetted soil volume under an emitter. The partial soil wetting pattern by micro irrigation requires assessment of the percentage of soil volume that is wetted (Moshe. 2006). The distance between emitter on lateral pipe and distance of lateral pipes from each other should be determined based on the degree of wetted soil diameter by emitters. The duration of irrigation also depends on the fact that at what time after commencement of irrigation, the wetting front reaches depth of plant's root or a multiple of it. The distance of outlets, discharge rate and time of irrigation in drip irrigation have to be determined so that volume of wetted soil is close to volume of plant's root as much as possible. The volume of wetted soil surface and moisture in onion shape depends on different factors including soil texture and layering, soil homogeneity, dripper flow rate, primary moisture of soil, consumption water and land slope. Analyses on the effects of application rate on the water distribution pattern demonstrated that increasing the water application rate allows more water to distribute in horizontal direction, while decreasing the rate allows more water to distribute in vertical direction for a given volume applied (Li et al., 2004).

Although papaya is generally considered to be drought sensitive and responsive to irrigation, there is a shortage of good experimental evidence to support this view. There is a need to establish practical irrigation schedules for this remarkable crop. Further, to enhance the productivity of the crop, optimum plant population is very crucial. When spacing is varied, it further warrants for understanding the requirement of wetted volume for standardizing the drip irrigation practice in a given agro-climatic condition. Keeping this in view, field experiments were conducted to adjudge an optimumsoil volume wetting in papaya along with different plant spacing.

\section{MATERIALS AND METHODS}

Field experiments were conducted during 2015 to 2019 at ICAR- Indian Institute of Horticultural Research, Hessaraghatta, Bengaluru located at a latitude of $13^{\circ} 8^{\prime} 12^{\prime \prime} \mathrm{N}$ and a longitude of $77^{\circ} 29^{\prime} 45^{\prime \prime} \mathrm{E}$. The experimental soil was sandy loam in texture with a $\mathrm{pH}$ of 6.14 and an EC of $0.067 \mathrm{dSm}^{-1}$. The maximum temperature during the experimental period ranged from $24^{\circ} \mathrm{C}$ to $36^{\circ} \mathrm{C}$ and the minimum temperature ranged between $10^{\circ} \mathrm{C}$ to $22^{\circ} \mathrm{C}$. The period between March to May are the warm months with higher temperatures and evaporation while the period between November to January were the cooler months with low temperature and evaporation. The average relative humidity was higher during September and October months. The average rainfall of the region is around $850 \mathrm{~mm}$ with two peak periods of rainfall during June- July and September- October months. Pre-experimental soil had a $\mathrm{pH}$ of 6.32 with almost no salts $\left(0.14 \mathrm{dSm}^{-1}\right)$. The organic carbon content of the soil was quite low $(0.15 \%)$. Although the available nitrogen content of the soil was low (56 $\mathrm{kg} / \mathrm{ha})$, the available phosphorus was higher $(51.5 \mathrm{~kg} /$ ha). Further, the available potassium content of the soil was also higher (315 kg/ha).

Field experiments were conducted in papaya (Cv. Red Lady) in split plot design with three plant spacing $(1.5 \times 1.5 \mathrm{~m} ., 1.8 \times 1.5 \mathrm{~m}$. and $1.8 \times 1.8 \mathrm{~m}$.) as main plot treatments and three levels of soil volume wetting (30\%. 50\% and $70 \%)$ as sub plot treatments in four replications. The crop was raised with recommended package of practices except for irrigation. Irrigation was scheduled based on pan evaporation and the per cent soil volume was restricted by inserting a barrier in the root zone. The calculated amount of water for each irrigation was either partially wetted or fully wetted in the root zone depending on the treatment. All the growth and yield parameters were recorded in both the crops. The root length and depth were recorded based on longest horizontal and vertical growth, respectively and the root volume was measured based on the quantity of water displaced by immersing in water. The root dry weight was recorded by carefully collecting the roots and drying in hot air oven till constant weight was obtained. All the mean data was analyzed as per standard statistical procedures (Panse and Sukhatme, 1985).

\section{RESULTS AND DISCUSSION}

\section{Plant Growth}

Although majority of the growth parameters showed non-significant differences among different per cent soil volume wetting irrigation levels, plant spacing was found to affect the plant stature, leaf production and height at first flowering significantly (Table 1). Giving wider spacing of $1.8 \mathrm{~m} \times 1.8 \mathrm{~m}$ for papaya was found to favour the crop growth through higher plant height 
$(1.57 \mathrm{~m})$ significantly differing from both $1.8 \mathrm{~m} \mathrm{x}$ $1.5 \mathrm{~m}$ and $1.5 \mathrm{~m} \times 1.5 \mathrm{~m}$ plant spacing. The leaf production followed a similar trend with significantly higher leaf production at wider spacing of $1.8 \mathrm{~m} \mathrm{x} 1.8$ $\mathrm{m}$ (24.5 leaves/plant). It is worth to note that narrowing the plant rows drastically reduced the plant height while leaf production affected much due to reduction in intra row spacing. This assumes significance in papaya as source sink balance is critical in papaya fruit set, development and sugar accumulation and in general each mature leaf can provide photo assimilate for about three fruits (Zhou et al., 2000). Further, the height at first fruiting was significantly lower with a spacing of $1.8 \mathrm{~m} \times 1.5 \mathrm{~m}$ $(56.4 \mathrm{~cm})$ significantly differing from both $1.5 \mathrm{~m} \mathrm{x}$ $1.5 \mathrm{~m}(60.9 \mathrm{~cm})$ or $1.8 \mathrm{~m} \mathrm{x} 1.8 \mathrm{~m}(66.8 \mathrm{~cm})$ plant spacing. Similar results were reported in papaya by Singh et al. (2010) wherein vegetative growth characters in papaya like plant height, numbers of leaves and inter-nodal length showed significant difference among all the treatments.

\section{Root growth parameters in papaya}

Root growth in general was significantly influenced both by the plant spacing as well asirrigation levels. Root length was significantly higher with $1.5 \mathrm{~m}$. x 1.5 $\mathrm{m}$ spacing $(109.8 \mathrm{~cm})$ ascompared to either $1.8 \mathrm{~m}$. $\mathrm{x}$ $1.8 \mathrm{~m} .(100.3 \mathrm{~cm})$ or $1.8 \mathrm{~m}$. x $1.5 \mathrm{~m}$. $(91.9 \mathrm{~cm})$. Among the irrigation levels, meeting $50 \%$ ER and irrigating in part of the root zone $(50 \%)$ was found to have higher root length $(114.7 \mathrm{~cm})$ significantly differing from others. Further, the interaction between spacing and irrigation levels was significant with highest root length $(126.7 \mathrm{~cm})$ recording in $1.5 \mathrm{~m} \mathrm{x} 1.5 \mathrm{~m}$ spacing with $50 \%$ ER irrigating in $50 \%$ of the root zone (Table 2).

The rooting depth in papaya was influenced significantly both by plant spacing and interaction of spacing with irrigation levels. Planting at a distance of $1.8 \mathrm{~m} \times 1.5 \mathrm{~m}$ was found to produce significantly deeper roots $(54.2 \mathrm{~cm})$ over other spacings and among the interactions, planting at $1.8 \mathrm{~m} \times 1.5 \mathrm{~m}$ and irrigation meeting $30 \%$ of ER wetting $100 \%$ of the root volume resulted in significantly higher rooting depth $(61.7 \mathrm{~cm})$.

The root volume in general differed significantly both with the plant spacing and the irrigation levels.
Closer planting at $1.5 \mathrm{~m} \times 1.5 \mathrm{~m}$ distance had shown significantly higher root volume $\left(2148 \mathrm{~cm}^{3}\right)$ as compared to either $1.8 \mathrm{~m} \mathrm{x} 1.5 \mathrm{~m}\left(1983 \mathrm{~cm}^{3}\right)$ or $1.5 \mathrm{~m}$ $\mathrm{x} 1.5 \mathrm{~m}\left(1671 \mathrm{~cm}^{3}\right)$. Among the irrigation levels, meeting $70 \%$ ER and irrigating in part of the root zone $(70 \%)$ was found to have higher root volume $\left(2572 \mathrm{~cm}^{3}\right)$ significantly differing from others. Further, the interaction between spacing and irrigation levels was significant with highest root volume $\left(2833.3 \mathrm{~cm}^{3}\right)$ observed under $1.8 \mathrm{~m} \mathrm{x} 1.5 \mathrm{~m}$ spacing with irrigation meeting $70 \% \mathrm{ER}$ and wetting $70 \%$ of the root zone.

The oven dry weight of roots was significantly influenced by plant spacing. The significantly higher oven dry weight of root in papaya was observed under plant spacing of $1.5 \mathrm{~m} \mathrm{x} 1.5 \mathrm{~m}$ (516.7 g/plant) as compared to either $1.8 \mathrm{~m} \mathrm{x} 1.5 \mathrm{~m}(237.2 \mathrm{~g} /$ plant $)$ or $1.8 \mathrm{~m} \mathrm{x} 1.5 \mathrm{~m}(279.6 \mathrm{~g} / \mathrm{plant})$ which may be attributed to the higher growth of roots in competing environments in search of resources at closer spacing with higher intra-plant competitions. Wang et al. (2006) also reported that root development and distribution are affected by spatial and temporal soil water distribution.

\section{Photosynthetic rate}

The photosynthetic response of papaya is strongly linked to environmental conditions through stomatal behavior (Zhou et al., 2004). The photosynthetic rate recorded in papaya in different treatment combinations is depicted in Fig.1. It was observed that the spacing of papaya plant to a distance of $1.8 \mathrm{~m} \times 1.8 \mathrm{~m}$ influenced the photosynthetic rate $\left(10.85 \mu \mathrm{mol} / \mathrm{m}^{2} / \mathrm{s}\right)$ significantly as compared to $1.5 \mathrm{~m} . \times 1.5 \mathrm{~m}$. $\left(9.09 \mu \mathrm{mol} / \mathrm{m}^{2} / \mathrm{s}\right)$ although it was at par $_{2}$ with the spacing of $1.8 \mathrm{~m}$. x $1.5 \mathrm{~m}$. $(9.48 \mu$ $\mathrm{mol} / \mathrm{m} / \mathrm{s}$ ). Among the irrigation levels, meeting $70 \%$ of the ER and wetting $70 \%$ soil volume recorded the highest photosynthetic rate $(10.52 \mu$ $\mathrm{mol} / \mathrm{m}^{2} / \mathrm{s}$ ) which was found to differ significantly with $50 \%$ of the ER wetting $50 \%$ of the soil volume $\left(8.41 \mu \mathrm{mol} / \mathrm{m}^{2} / \mathrm{s}\right)$. Although the interaction effects were not significant, the highest photosynthetic rate of $11.73 \mu \mathrm{mol} / \mathrm{m}^{2} / \mathrm{s}$ was recorded with $1.8 \mathrm{~m} \mathrm{x}$ $1.8 \mathrm{~m}$ spacing at $70 \%$ ER irrigation wetting $70 \%$ soil volume. Santas et al., (2015) also affirmed that the fruit production and physiological characteristics of papaya depend on planting spacing. 
Table 1. Growth attributes in papaya as influenced by spacing and soil volume wetting irrigation treatments

\begin{tabular}{|c|c|c|c|c|c|c|c|}
\hline \multirow[t]{2}{*}{ Spacing } & \multirow[t]{2}{*}{ Irrigation level } & \multirow[t]{2}{*}{$\begin{array}{l}\text { Plant } \\
\text { height } \\
(\mathrm{cm})\end{array}$} & \multirow[t]{2}{*}{$\begin{array}{l}\text { Collar } \\
\text { girth } \\
(\mathrm{cm})\end{array}$} & \multirow[t]{2}{*}{$\begin{array}{c}\text { No. of } \\
\text { leaves/ } \\
\text { plant }\end{array}$} & \multicolumn{2}{|c|}{$\begin{array}{l}\text { Canopy } \\
\text { spread } \\
(\mathrm{m})\end{array}$} & \multirow[t]{2}{*}{$\begin{array}{l}\text { Height at first } \\
\text { fruiting } \\
(\mathrm{cm})\end{array}$} \\
\hline & & & & & E-W & $\mathbf{N}-\mathrm{S}$ & \\
\hline \multirow[t]{5}{*}{$1.5 \mathrm{~m} \times 1.5 \mathrm{~m}$} & $\begin{array}{c}\text { Irrigation at } 30 \% \text { ER } \\
\text { wetting } 30 \% \text { soil } \\
\text { volume }\end{array}$ & 133.0 & 24.3 & 17.4 & 142.0 & 147.0 & 62.8 \\
\hline & $\begin{array}{c}\text { Irrigation at } 50 \% \text { ER } \\
\text { wetting } 50 \% \text { soil } \\
\text { volume }\end{array}$ & 140.0 & 26.4 & 20.0 & 154.0 & 168.0 & 58.6 \\
\hline & $\begin{array}{c}\text { Irrigation at } 70 \% \text { ER } \\
\text { wetting } 70 \% \text { soil } \\
\text { volume }\end{array}$ & 143.0 & 28.0 & 21.4 & 154.0 & 156.5 & 58.0 \\
\hline & $\begin{array}{c}\text { Irrigation at } 70 \% \text { ER } \\
\text { wetting } 100 \% \text { soil } \\
\text { volume }\end{array}$ & 137.7 & 26.1 & 17.5 & 167.0 & 138.7 & 64.0 \\
\hline & Mean & 138.4 & 26.2 & 19.1 & 154.3 & 152.6 & 60.9 \\
\hline \multirow[t]{5}{*}{$1.8 \mathrm{~m} \times 1.5 \mathrm{~m}$} & $\begin{array}{c}\text { Irrigation at } 30 \% \text { ER } \\
\text { wetting } 30 \% \text { soil } \\
\text { volume }\end{array}$ & 132.0 & 26.6 & 24.4 & 146.0 & 144.0 & 61.8 \\
\hline & $\begin{array}{c}\text { Irrigation at } 50 \% \text { ER } \\
\text { wetting } 50 \% \text { soil } \\
\text { volume }\end{array}$ & 146.0 & 28.2 & 23.8 & 165.0 & 156.0 & 55.0 \\
\hline & $\begin{array}{c}\text { Irrigation at } 70 \% \text { ER } \\
\text { wetting } 70 \% \text { soil } \\
\text { volume }\end{array}$ & 138.0 & 26.8 & 22.4 & 154.0 & 152.0 & 54.0 \\
\hline & $\begin{array}{c}\text { Irrigation at } 70 \% \text { ER } \\
\text { wetting } 100 \% \text { soil } \\
\text { volume }\end{array}$ & 143.0 & 24.7 & 22.5 & 150.3 & 152.7 & 54.8 \\
\hline & Mean & 139.8 & 26.6 & 23.3 & 153.8 & 151.2 & 56.4 \\
\hline \multirow[t]{7}{*}{$1.8 \mathrm{~m} \times 1.8 \mathrm{~m}$} & $\begin{array}{c}\text { Irrigation at } 30 \% \text { ER } \\
\text { wetting } 30 \% \text { soil } \\
\text { volume }\end{array}$ & 167.0 & 28.0 & 25.8 & 162.0 & 161.0 & 66.8 \\
\hline & $\begin{array}{c}\text { Irrigation at } 50 \% \text { ER } \\
\text { wetting } 50 \% \text { soil } \\
\text { volume }\end{array}$ & 140.0 & 25.0 & 21.6 & 154.0 & 158.0 & 66.4 \\
\hline & $\begin{array}{c}\text { Irrigation at } 70 \% \text { ER } \\
\text { wetting } 70 \% \text { soil } \\
\text { volume }\end{array}$ & 162.0 & 28.2 & 21.8 & 163.0 & 161.0 & 69.4 \\
\hline & $\begin{array}{c}\text { Irrigation at } 70 \% \text { ER } \\
\text { wetting } 100 \% \text { soil } \\
\text { volume }\end{array}$ & 159.0 & 29.2 & 28.7 & 169.7 & 174.8 & 64.7 \\
\hline & Mean & 157.0 & 27.4 & 24.5 & 162.2 & 163.7 & 66.8 \\
\hline & $\begin{array}{l}\text { S.Em } \pm \\
\quad \text { Main } \\
\text { Sub } \\
\text { Main x Sub }\end{array}$ & $\begin{array}{l}2.47 \\
2.65 \\
4.93\end{array}$ & $\begin{array}{l}0.60 \\
0.73 \\
1.19\end{array}$ & $\begin{array}{l}0.84 \\
1.17 \\
1.68\end{array}$ & $\begin{array}{l}3.10 \\
4.18 \\
6.20\end{array}$ & $\begin{array}{c}5.874 \\
6.559 \\
11.748\end{array}$ & $\begin{array}{l}1.19 \\
1.52 \\
2.39\end{array}$ \\
\hline & 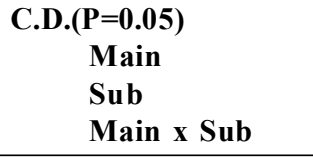 & $\begin{array}{c}8.17 \\
\mathrm{NS} \\
13.75\end{array}$ & $\begin{array}{l}\text { NS } \\
\text { NS } \\
\text { NS }\end{array}$ & $\begin{array}{c}2.79 \\
\mathrm{NS} \\
\mathrm{NS}\end{array}$ & $\begin{array}{l}\text { NS } \\
\text { NS } \\
\text { NS }\end{array}$ & $\begin{array}{l}\text { NS } \\
\text { NS } \\
\text { NS }\end{array}$ & $\begin{array}{c}3.95 \\
\mathrm{NS} \\
\mathrm{NS}\end{array}$ \\
\hline
\end{tabular}


Standardization of spacing and soil volume wetting for drip irrigation in papaya

Table 2. Root growthin papaya as influenced by plant spacing and irrigation levels

\begin{tabular}{|c|c|c|c|c|c|}
\hline Spacing & Irrigation level & $\begin{array}{l}\text { Root length } \\
\quad(\mathrm{cm})\end{array}$ & $\begin{array}{l}\text { Root depth } \\
\quad(\mathrm{cm})\end{array}$ & $\begin{array}{l}\text { Root volume } \\
\qquad\left(\mathrm{cm}^{3}\right)\end{array}$ & $\begin{array}{l}\text { Dry weight of } \\
\text { roots }(\mathrm{g} / \text { plant })\end{array}$ \\
\hline \multirow[t]{5}{*}{$\begin{array}{l}1.5 \mathrm{~m} \\
\mathrm{x} 1.5 \mathrm{~m}\end{array}$} & $\begin{array}{c}\text { Irrigation at } 30 \% \\
\text { ER wetting } 30 \% \text { soil volume }\end{array}$ & 110.0 & 56.7 & 2866.7 & 688.3 \\
\hline & $\begin{array}{c}\text { Irrigation at } 50 \% \text { ER } \\
\text { wetting } 50 \% \text { soil volume }\end{array}$ & 126.7 & 48.3 & 2000.0 & 356.2 \\
\hline & $\begin{array}{c}\text { Irrigation at } 70 \% \text { ER } \\
\text { wetting } 70 \% \text { soil volume }\end{array}$ & 102.5 & 52.5 & 2800.0 & 540.7 \\
\hline & $\begin{array}{c}\text { Irrigation at } 70 \% \text { ER } \\
\text { wetting } 100 \% \text { soil volume }\end{array}$ & 100.0 & 42.5 & 925.0 & 481.5 \\
\hline & Mean & 109.8 & 50.0 & 2147.9 & 516.7 \\
\hline \multirow[t]{5}{*}{$\begin{array}{r}1.8 \mathrm{~m} \\
\mathrm{x} 1.5 \mathrm{~m}\end{array}$} & $\begin{array}{c}\text { Irrigation at } 30 \% \text { ER } \\
\text { wetting } 30 \% \text { soil volume }\end{array}$ & 91.7 & 61.7 & 2350.0 & 317.4 \\
\hline & $\begin{array}{c}\text { Irrigation at } 50 \% \text { ER } \\
\text { wetting } 50 \% \text { soil volume }\end{array}$ & 100.0 & 46.7 & 1850.0 & 232.7 \\
\hline & $\begin{array}{c}\text { Irrigation at } 70 \% \text { ER } \\
\text { wetting } 70 \% \text { soil volume }\end{array}$ & 103.3 & 50.0 & 2833.3 & 299.5 \\
\hline & $\begin{array}{c}\text { Irrigation at } 70 \% \text { ER } \\
\text { wetting } 100 \% \text { soil volume }\end{array}$ & 72.5 & 58.5 & 900.0 & 99.2 \\
\hline & Mean & 91.9 & 54.2 & 1983.3 & 237.2 \\
\hline \multirow[t]{13}{*}{$\begin{array}{l}1.8 \mathrm{~m} \\
\mathrm{x} 1.8 \mathrm{~m}\end{array}$} & $\begin{array}{c}\text { Irrigation at } 30 \% \text { ER } \\
\text { wetting } 30 \% \text { soil volume }\end{array}$ & 75.0 & 40.0 & 800.0 & 171.3 \\
\hline & $\begin{array}{c}\text { Irrigation at } 50 \% \text { ER } \\
\text { wetting } 50 \% \text { soil volume }\end{array}$ & 117.5 & 45.0 & 2000.0 & 368.5 \\
\hline & $\begin{array}{c}\text { Irrigation at } 70 \% \text { ER } \\
\text { wetting } 70 \% \text { soil volume }\end{array}$ & 111.3 & 43.3 & 2083.3 & 337.6 \\
\hline & $\begin{array}{c}\text { Irrigation at } 70 \% \text { ER } \\
\text { wetting } 100 \% \text { soil volume }\end{array}$ & 97.5 & 45.0 & 1800.0 & 241.1 \\
\hline & Mean & 100.3 & 43.3 & 1670.8 & 279.6 \\
\hline & S.Em \pm & & & & \\
\hline & Main & 1.87 & 2.18 & 98.49 & 48.00 \\
\hline & Sub & 3.06 & 1.85 & 119.55 & 61.09 \\
\hline & Main x Sub & 4.96 & 3.53 & 204.60 & 103.45 \\
\hline & C.D. $(P=0.05)$ & & & & \\
\hline & Main & 6.59 & 7.68 & 347.46 & 169.33 \\
\hline & Sub & 8.93 & NS & 348.76 & NS \\
\hline & Main x Sub & 14.89 & 11.11 & 625.80 & NS \\
\hline
\end{tabular}




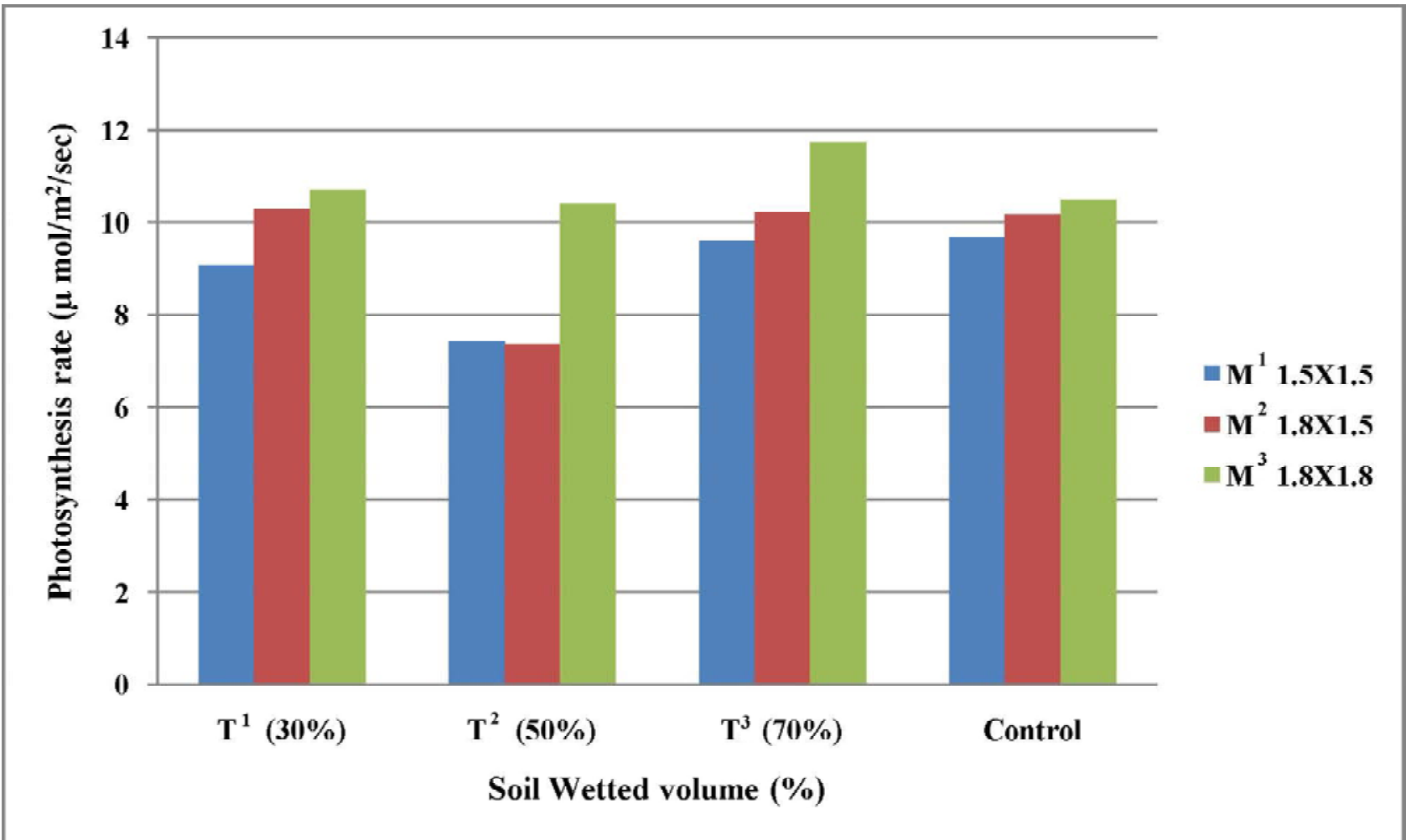

Fig 1. Effect of different spacing and soil volume wetting irrigation on photosynthetic rate in papaya

\section{Fruit yield}

Although the mean fruit number per plant not affected significantly by plant spacing, different levels of soil volume wetting irrigation had a significant influence (Table 3). Irrigating the plant to wet $70 \%$ of the soil volume resulted in significantly higher number of fruits (17.2/plant) as compared to $30 \%(10.6 /$ plant $)$ and normal drip irrigation (13.3/plant) although it was on par with $50 \%$ of the soil volume (14.8/plant). Although the fruit yield in general was slightly lower in the crop owing to the incidence of PRSV disease (even with sufficient care to combat the disease), the treatment effects were very clear both for the spacing and the irrigation levels.

Significantly higher mean fruit yield (42.2 $\mathrm{t} / \mathrm{ha}$ ) was recorded with the spacing of $1.5 \mathrm{~m} \times 1.5 \mathrm{~m}$ as compared to either $1.8 \mathrm{~m} \times 1.5 \mathrm{~m}(23.4 \mathrm{t} / \mathrm{ha})$ or $1.8 \mathrm{~m}$ $x 1.8 \mathrm{~m}(22.1 \mathrm{t} / \mathrm{ha})$. The mean increase in yield on the basis of two crops ranged from 80.3 per cent (over $1.8 \times 1.5 \mathrm{~m}$ spacing) to 91.0 per cent (over $1.8 \mathrm{~m} \mathrm{x}$ $1.8 \mathrm{~m}$ spacing). This increased fruit yield at closer spacing of $1.5 \mathrm{~m} \times 1.5 \mathrm{~m}$ was not only due to more number of plants per unit area but also was due to higher number of fruits (16.4/plant) over other spacings. Although different soil volume wetting irrigation failed to affect the mean fruit yield significantly, irrigation at 70 per cent ER and wetting either $70 \%$ of soil volume $(31.7 \mathrm{t} / \mathrm{ha})$ or $100 \%$ of soil volume $(31.2 \mathrm{t} / \mathrm{ha})$ showed higher fruit yield. The response in total yield to the irrigation applied was quadratic and increasing in the range to the amounts of water applied from 30, through 50 to 70 per cent of the ER. Similar results of higher yield with increasing trend in irrigation levels each year was also reported earlier in orange by Petillo (2004).

Significantly higher water use efficiency $(71.3 \mathrm{~kg} /$ ha.mm) was recorded in papaya by following closer spacing of $1.5 \mathrm{~m} \mathrm{x} 1.5 \mathrm{~m}$ which decreased drastically with increase in spacing either to $1.8 \mathrm{~m} \mathrm{x} 1.8 \mathrm{~m}$ $(34.6 \mathrm{~kg} / \mathrm{ha} . \mathrm{mm})$ or $1.8 \mathrm{~m} \times 1.5 \mathrm{~m}(37.3 \mathrm{~kg} /$ ha.mm).This suggests that under limited water situations following an ideal agronomic package is also essential to get more output per unit amount of water used. 
Standardization of spacing and soil volume wetting for drip irrigation in papaya

Table 3. Fruit yield and water use efficiency in papaya as influenced by spacing and soil volume wetting in two crops of papaya

\begin{tabular}{|c|c|c|c|c|c|c|c|c|c|c|c|c|c|}
\hline \multirow[t]{2}{*}{ Spacing } & \multirow[t]{2}{*}{$\begin{array}{l}\text { Soil volume } \\
\text { wetting }(\%)\end{array}$} & \multicolumn{3}{|c|}{ No. of fruits /plant } & \multicolumn{3}{|c|}{ Fruit yield / plant (kg) } & \multicolumn{3}{|c|}{ Papaya yield (t/ha) } & \multicolumn{3}{|c|}{$\begin{array}{l}\text { Water use efficiency } \\
\text { (kg/ha.mm) }\end{array}$} \\
\hline & & $\begin{array}{l}2016- \\
17\end{array}$ & $\begin{array}{l}2018- \\
19\end{array}$ & Mean & $\begin{array}{l}2016- \\
17\end{array}$ & $\begin{array}{l}2018- \\
19\end{array}$ & Mean & $\begin{array}{l}2016- \\
17\end{array}$ & $\begin{array}{l}2018- \\
19\end{array}$ & Mean & $\begin{array}{l}2016- \\
17\end{array}$ & $\begin{array}{l}2018- \\
19\end{array}$ & Mean \\
\hline \multirow[t]{5}{*}{$\begin{array}{l}1.5 \mathrm{~m} \times \\
1.5 \mathrm{~m}\end{array}$} & $\begin{array}{l}\text { Irrigation at } 30 \% \text { ER } \\
\text { wetting } 30 \% \text { root } \\
\text { zone volume }\end{array}$ & 13.00 & 19.90 & 16.50 & 10.30 & 10.40 & 10.30 & 45.6 & 46.03 & 45.79 & 110.08 & 148.01 & 129.04 \\
\hline & $\begin{array}{l}\text { Irrigation at } 50 \% \text { ER } \\
\text { wetting } 50 \% \text { root } \\
\text { zone volume }\end{array}$ & 11.30 & 20.40 & 15.90 & 8.00 & 8.77 & 8.38 & 35.6 & 39.70 & 37.63 & 51.55 & 74.88 & 63.21 \\
\hline & $\begin{array}{l}\text { Irrigation at } 70 \% \mathrm{ER} \\
\text { wetting } 70 \% \text { root } \\
\text { zone volume }\end{array}$ & 16.90 & 19.90 & 18.40 & 8.95 & 10.40 & 9.66 & 39.8 & 34.90 & 37.30 & 40.92 & 52.57 & 46.75 \\
\hline & Normal drip irrigation & 18.10 & 11.30 & 14.70 & 11.2 & 5.84 & 8.53 & 49.9 & 46.10 & 47.98 & 62.69 & 29.62 & 46.15 \\
\hline & Mean & 14.80 & 17.90 & 16.40 & 9.61 & 8.83 & 9.22 & 42.7 & 41.7 & 42.20 & 66.31 & 76.27 & 71.29 \\
\hline \multirow[t]{5}{*}{$\begin{array}{l}1.8 \mathrm{~m} \times \\
1.5 \mathrm{~m}\end{array}$} & $\begin{array}{l}\text { Irrigation at } 30 \% \text { ER } \\
\text { wetting } 30 \% \\
\text { root zone volume }\end{array}$ & 9.70 & 8.63 & 9.15 & 5.95 & 4.46 & 5.21 & 22.05 & 16.50 & 19.30 & 53.29 & 53.14 & 53.21 \\
\hline & $\begin{array}{l}\text { Irrigation at } 50 \% \text { ER } \\
\text { wetting } 50 \% \text { root } \\
\text { zone volume }\end{array}$ & 13.70 & 12.60 & 13.10 & 6.38 & 6.75 & 6.56 & 23.60 & 25.00 & 24.30 & 34.25 & 48.04 & 41.15 \\
\hline & $\begin{array}{l}\text { Irrigation at } 70 \% \mathrm{ER} \\
\text { wetting } 70 \% \text { root } \\
\text { zone volume }\end{array}$ & 9.70 & 20.50 & 15.10 & 3.90 & 11.2 & 7.54 & 14.50 & 41.40 & 27.90 & 14.96 & 47.25 & 31.10 \\
\hline & Normal drip irrigation & 11.60 & 11.70 & 11.60 & 6.40 & 5.48 & 5.94 & 23.70 & 20.30 & 21.99 & 24.53 & 23.15 & 23.84 \\
\hline & Mean & 11.20 & 13.30 & 12.20 & 5.66 & 6.97 & 6.31 & 20.96 & 25.80 & 23.40 & 31.75 & 42.89 & 37.32 \\
\hline \multirow[t]{13}{*}{$\begin{array}{l}1.8 \mathrm{~m} \times \\
1.8 \mathrm{~m}\end{array}$} & $\begin{array}{l}\text { Irrigation meeting } \\
30 \% \text { ER wetting } 30 \% \\
\text { soil volume }\end{array}$ & 3.43 & 8.68 & 6.05 & 1.60 & 4.57 & 3.09 & 4.93 & 14.10 & 9.51 & 11.90 & 45.35 & 28.63 \\
\hline & $\begin{array}{l}\text { Irrigation meeting } \\
50 \% \text { ER wetting } 50 \% \\
\text { soil volume }\end{array}$ & 10.18 & 20.40 & 15.28 & 5.30 & 11.20 & 8.25 & 16.38 & 34.50 & 25.50 & 35.60 & 66.38 & 50.99 \\
\hline & $\begin{array}{l}\text { Irrigation meeting } \\
70 \% \text { ER wetting } 70 \% \\
\text { soil volume }\end{array}$ & 12.40 & 23.50 & 17.96 & 8.58 & 10.70 & 9.66 & 26.45 & 33.20 & 29.80 & 27.38 & 37.85 & 32.61 \\
\hline & $\begin{array}{l}\text { Irrigation meeting } \\
70 \% \text { ER and wetting } \\
100 \% \text { soil volume }\end{array}$ & 10.90 & 16.40 & 13.70 & 6.35 & 9.02 & 7.69 & 19.60 & 27.80 & 23.70 & 20.28 & 31.78 & 26.03 \\
\hline & Mean & 9.23 & 17.24 & 13.20 & 5.46 & 8.88 & 7.17 & 16.80 & 27.40 & 22.10 & 23.79 & 45.34 & 34.57 \\
\hline & S.Em \pm & & & & & & & & & & & & \\
\hline & Main & 1.54 & 1.75 & 1.26 & 0.86 & 1.09 & 0.85 & 3.79 & 4.06 & 3.46 & 4.44 & 6.86 & 4.82 \\
\hline & Sub & 0.97 & 1.81 & 1.02 & 0.77 & 1.00 & 0.63 & 3.09 & 3.84 & 2.54 & 6.19 & 8.47 & 5.40 \\
\hline & Main x Sub ${ }^{-1}$ & 2.12 & 3.23 & 1.98 & 1.45 & 1.86 & 1.23 & 5.99 & 7.05 & 5.15 & 10.30 & 14.43 & 9.43 \\
\hline & C.D $(P=0.05)$ & & & & & & & & & & & & \\
\hline & Main & NS & NS & NS & 3.05 & NS & NS & 13.38 & NS & 12.20 & 15.6 & 24.2 & 17.00 \\
\hline & Sub & 2.82 & 5.29 & 2.97 & NS & 2.93 & 1.85 & NS & NS & NS & 18.0 & 24.7 & 15.80 \\
\hline & Main x Sub ${ }^{-1}$ & 6.86 & NS & NS & 4.53 & NS & 4.05 & NS & NS & NS & 31.2 & 44.1 & 29.00 \\
\hline
\end{tabular}


Among the interactions, higher papaya yield (48.0 $\mathrm{t}$ / ha) was recorded with normal drip irrigation $(80 \%$ soil volume wetting) under closer spacing $(1.5 \mathrm{~m} \mathrm{x} 1.5 \mathrm{~m})$. However, higher water use efficiency $(129 \mathrm{~kg} /$ ha.mm) could be obtained by scheduling the irrigation at $30 \%$ soil volume wetting especially by planting at $1.5 \times 1.5 \mathrm{~m}$ spacing.

\section{The economics of papaya cultivation}

The economics of growing papaya under different spacings with irrigation levels is presented in Table 4. It was observed that the cost of production of papaya was although 33 per cent higher under closer spacing of $1.5 \mathrm{~m} \times 1.5 \mathrm{~m}$ (Rs.3,69,400/ha), the gross returns were also significantly higher
(Rs.6,32,740/ha). The higher cost of production with closer spacings may be attributed to the additional investment cost on planting material, pit making and other inputs like manures and fertilizers. Sagar et al., (2012) also found that papaya was highly capital intensive crop and average cost of cultivation per hectare was Rs.176660. The higher gross returns with closer spacing may be attributed not only to the more number of yielding plants but also higher yield per plant. Higher net returns were recorded with closer spacing of $1.5 \mathrm{~m} \mathrm{x} 1.5 \mathrm{~m}$ (Rs.2,63,290/ha). Further, benefit cost ratio was although higher (1.72) with closer spacing, the results were found to be non-significant.

Table 4. The mean economics of papaya cultivation under different spacing and irrigation levels

\begin{tabular}{|c|c|c|c|c|c|}
\hline Spacing & Irrigation level & $\begin{array}{c}\text { Cost of } \\
\text { production } \\
\text { (Rs/ha) }\end{array}$ & $\begin{array}{l}\text { Gross } \\
\text { returns } \\
\text { (Rs/ha) }\end{array}$ & $\begin{array}{c}\text { Net } \\
\text { returns } \\
\text { (Rs/ha) }\end{array}$ & $\begin{array}{l}\text { B:C } \\
\text { ratio }\end{array}$ \\
\hline \multirow[t]{5}{*}{$1.5 \mathrm{~m} \times 1.5 \mathrm{~m}$} & $\begin{array}{l}\text { Irrigation at 30\% ER wetting 30\% } \\
\text { root zone volume }\end{array}$ & $3,47,700$ & $6,86,900$ & $3,39,020$ & 1.98 \\
\hline & $\begin{array}{l}\text { Irrigation at } 50 \% \text { ER wetting } 50 \% \\
\text { root zone volume }\end{array}$ & $3,65,060$ & $5,64,400$ & $1,99,340$ & 1.55 \\
\hline & $\begin{array}{l}\text { Irrigation at } 70 \% \text { ER wetting } 70 \% \\
\text { root zone volume }\end{array}$ & $3,82,430$ & $5,59,980$ & $1,77,550$ & 1.46 \\
\hline & Normal drip irrigation & $3,82,430$ & $7,19,670$ & $3,37,240$ & 1.88 \\
\hline & Mean & $3,69,400$ & $6,32,740$ & $2,63,290$ & 1.72 \\
\hline \multirow[t]{5}{*}{$1.8 \mathrm{~m} \mathrm{x1.5 \textrm {m }}$} & $\begin{array}{l}\text { Irrigation at } 30 \% \text { ER wetting } 30 \% \\
\text { root zone volume }\end{array}$ & $2,97,810$ & $3,91,740$ & 93,930 & 1.31 \\
\hline & $\begin{array}{l}\text { Irrigation at } 50 \% \text { ER wetting } 50 \% \\
\text { root zone volume }\end{array}$ & $3,15,180$ & $3,64,690$ & 49,510 & 1.16 \\
\hline & $\begin{array}{l}\text { Irrigation at } 70 \% \text { ER wetting } 70 \% \\
\text { root zone volume }\end{array}$ & $3,32,550$ & $4,18,790$ & 86,240 & 1.26 \\
\hline & Normal drip irrigation & $3,32,550$ & $3,91,740$ & 93,930 & 1.31 \\
\hline & Mean & $3,19,520$ & $3,91,740$ & 80,900 & 1.26 \\
\hline \multirow[t]{5}{*}{$1.8 \mathrm{~m} \mathrm{x1.8 \textrm {m }}$} & $\begin{array}{l}\text { Irrigation at } 30 \% \text { ER wetting } 30 \% \\
\text { root zone volume }\end{array}$ & $2,55,970$ & $3,94,910$ & $1,38,940$ & 1.54 \\
\hline & $\begin{array}{l}\text { Irrigation at } 50 \% \text { ER wetting } 50 \% \\
\text { root zone volume }\end{array}$ & $2,73,340$ & $3,81,880$ & $1,08,540$ & 1.4 \\
\hline & $\begin{array}{l}\text { Irrigation at } 70 \% \text { ER wetting } 70 \% \\
\text { root zone volume }\end{array}$ & $2,90,710$ & $4,47,040$ & $1,56,330$ & 1.54 \\
\hline & Normal drip irrigation & $2,90,710$ & $3,55,820$ & 65,100 & 1.22 \\
\hline & Mean & $2,77,680$ & $3,94,920$ & $1,17,230$ & 1.43 \\
\hline
\end{tabular}


Among the irrigation levels, the gross returns were relatively higher (Rs. 4,91,180/ha) with irrigation at $30 \%$ ER and 30 per cent soil volume wetting, which may be attributed to the better soil moisture availability under the treatment in turn improving the productivity. Similarly, the net returns were also relatively higher with the treatment. Closer spacing of $1.5 \mathrm{~m} \times 1.5 \mathrm{~m}$ also recorded higher benefit cost ratio which may be attributed to both higher plant population (44\% higher) and the yield per plant (28\% higher). Higher benefit cost ratio with spacing of $1.5 \mathrm{~m} \mathrm{x} 1.5 \mathrm{~m}$ clearly indicated that it is worth to spend more for the inputs with closer spacing. Further, benefit cost ratio was relatively higher with 70 per cent soil volume wetting as compared to other irrigation levels.

\section{CONCLUSION}

The results of four years field trial in papaya on spacing and different soil volume based irrigation levels clearly indicated that under water scarcity conditions, it is worth irrigating papaya to meet only 30 per cent of the soil volume through a package of $1.5 \mathrm{~m} \times 1.5 \mathrm{~m}$ spacing so as to enhance the water use efficiency to the highest level $(129.04 \mathrm{~kg} / \mathrm{ha} . \mathrm{mm})$.

\section{ACKNOWLEDGEMENT}

The financial help rendered through sponsored research project on Consortia Research Platform on Water by Indian Council of Agricultural Research is gratefully acknowledged.

\section{REFERENCES}

Carr, M. K. V., 2014. The water relations and irrigation requirements of papaya (Carica papaya L.) : a review. Experimental Agri., 50 (2): 270-283.

Santas, E.M., Barbosa da Silva Jr., G., Cavalcante, I.H.L., Marques, A.S. and Albano, F.G. 2016. Planting spacing and $\mathrm{NK}$ fertilizing on physiological indexes and fruit production of papaya under semiarid climate, Bragantia 75

(1) Campinas Jan./Mar. 2016 Epub Dec 04, 2015, http://dx.doi.org/10.1590/1678-4499.111.

Eliemar Campostrini and David M.Glenn, 2007.

Ecophysiology of papaya- a review, Brazilian J. Plant Physiology, 19 (4) Online version ISSN 1677-9452, http://dx.doi.org/ 10.1590/S1677-04202007000400010

Petillo, G.M., Puppo, L., Chammorrow, A. and Hyashi, R. 2004. Effects of drip irrigation on the amount of water and wetted soil volume on Washington Navel orange yield, Proceedings of International Seminar on irrigation (Ed: R.C. Vallone), Acta Horti., 646 : 101-106.
Li, J., Zhang, J. and Rao, M. 2004. Wetting patterns and nitrogen distributions as affected by fertigation strategies from a surface point source. J.Agri. Water Management, 67 : 89104.

Panse, V.G. and Sukhatme, P.V. 1985. Statistical Methods for Agricultural Workers. Indian Council of Agricultural Research, New Delhi.

Sagar B., Parmar, H.C. and Darj V.B., 2012. Economics of production of papaya in middle Gujarat region of Gujarat, India, Global J. Bio, Agri. and Health Sci.,1(2):10-17.

Singh D.B., Roshan R.K., Pebam N., Yadav M., 2010. Effect of different spacings on growth, yield and yield characters of papaya (Carica papaya L.) cv. Coorg Honey Dew, Acta Horti., 851:291294.

Moshe, S. 2006. Micro irrigation in arid and semi-arid region Guidelines for planning and design. International Commission on Irrigation and drainage (ICID) 48 Nyaya Marg, Chanakyapuri, New Dehli-110 021, India. 
Wang H., Liu F., Andersen M.N., Jensen C.R., 2009. Comparative effects of partial root-zone drying and deficitirrigation on nitrogen uptake in potatoes (Solanum tuberosum L.). Irrigation Sci., 27: 443-447.
Zhou L, Christopher D.A., Paull R., 2000. Defoliation and fruit removal effects on papaya fruit production, sugar accumulation, and sucrose metabolism. J. American Soc. Horti. Sci., 125: 644-652.

(Received on 19.10.2019 and accepted on 21.03.2020) 